\title{
Modelling of a miniature mid-IR thermo-optic spectrometer on chip based on a $\mathrm{GaAs} / \mathrm{In}_{0.49} \mathrm{Ga}_{0.51} \mathrm{P}$ waveguide platform
}

\author{
Vasileios Mourgelas*, Neil P. Sessions, James S. Wilkinson and Ganapathy
}

Senthil Murugan

Optoelectronics Research Centre, University of Southampton, Southampton SO17 1BJ, UK

\section{ARTICLE INFO}

Keywords:

Mid-infrared

Spectroscopy

Thermo-optic

Semiconductors

MZI

Modelling

\begin{abstract}
Spectrometers based on integrated optical waveguides, as opposed to free space optics, provide many advantages including robustness, compactness, and low cost, combined with alignment-free and low-power operation. Interest in such a chip-based approach for integrated spectrometers is growing, but the integrated spectrometers demonstrated so far have largely been limited to operation at wavelengths up to about $5 \mu \mathrm{m}$ due to intrinsic material absorption. In this work, we design and model an onchip thermo-optically tuneable spectrometer based on a novel waveguide material platform consisting of GaAs (core) on InGaP (cladding), with potential to operate in the wavelength range between 1 and $17 \mu \mathrm{m}$ to fully cover the Mid-IR molecular fingerprint region. The strong thermo-optic effect exhibited by both of these materials makes the platform a very promising candidate for on-chip spectroscopy. A series of simulations were performed to predict the optical and thermal properties of the device. We show that with the proper optical and thermal optimisation of the platform and by using an interferometer with spiralled waveguides of $60 \mathrm{~mm}$ length, the device can effectively achieve a resolution of $10 \mathrm{~cm}^{-1}$ with a maximum temperature excursion of about $83 \mathrm{~K}$ and at the cost of a few tens of Watts of power.
\end{abstract}

\section{Introduction}

Optical spectroscopy using miniaturised devices has been extensively studied and several miniature spectrometers have been demonstrated in recent years [1-11]. Mid-IR (MIR) spectroscopy is of great importance in many different applications such as production monitoring, stand-off detection for security, environmental monitoring and biomedical diagnostics [12]. The reason behind its importance lies in the intrinsic selectivity present in the MIR spectral region $(2.5$ to $25 \mu \mathrm{m})$ in which all molecules display their characteristic fundamental vibrations. These vibrational "fingerprints" can be utilised in spectroscopic devices to produce a label free detection platform. Important attributes of a good miniature spectrometer are: 1) small footprint, 2) large refractive index (RI) tuning / high spectral resolution $\left(<10 \mathrm{~cm}^{-}\right.$ 1) 3) mass manufacturability using standardised fabrication techniques 4) high efficiency and 5) wide wavelength coverage.

Ultra-portable spectrometers operating in the near-infrared (NIR) region are commercially available [13], however,

*Corresponding author.

E-mail address: v.mourgelas@ soton.ac.uk (Vasileios Mourgelas). similar devices for the MIR region are at an early stage of development. NIR devices are far more convenient to fabricate and integrate within widely-deployed systems owing to more sensitive detectors, brighter sources and the more relaxed resolution requirements for condensed phase spectroscopy [13]. Despite their simplicity of design and fabrication, the application of NIR spectrometers to molecular spectroscopy is limited and compact MIR spectrometers are becoming essential. Portable MIR devices based on tuneable QCLs are just emerging; however, due to their high cost, large size compared to NIR spectrometers and the use of free space beams resulting in interference from atmospheric water absorption, alternative technological platforms are attractive [13]. One well-developed approach is to use MEMS to realise on-chip spectrometers. Although this method has been successfully applied to commercialised visible and NIR spectrometers, the spectral resolution of these devices is often very limited. This issue is very common in MEMS based devices and it is mostly related to mechanical tilting/air drag when the structures are operated using high energies/under atmospheric pressure $[6,13]$. One way to overcome this issue is to exploit the intrinsic properties of optical materials in 
tuneable waveguide-based devices. In mechanically-scanned devices, the scanning is performed by actuating physical mirrors to produce an optical path difference (OPD) whilst in waveguide-based electro-optically and thermo-optically tuneable devices the scanning is done by exposing one interferometer optical path to an external stimulus (e.g. electric field or heat). The main advantage of mechanically based compared to waveguide-based spectrometers, is their very broad potential spectral coverage due to free space propagation, which is not limited by the absorption of waveguide materials. Waveguide-based MIR spectrometers have been demonstrated in the past decade and are proving to be very promising [6-9].

Souza et al. [9] have presented an SOI FTIR thermo-optic spectrometer which achieved a resolution of $12.7 \mathrm{~cm}^{-1}$ for Cband erbium-doped fibre amplifier ASE input excitation. The device had a small footprint of the order of $1 \mathrm{~mm}^{2}$. The interferometer comprised of $\sim 30 \mathrm{~mm}$ spiralled arms. However, as $\mathrm{Si}$ suffers from strong optical absorption at wavelengths above $\lambda=6.5 \mu \mathrm{m}$ and $\mathrm{SiO}_{2}$ is highly absorptive above $\lambda=4 \mu \mathrm{m}$, the device was limited to shorter wavelengths within the MIR region. An alternative material platform that is widely being studied is based on $\mathrm{LiNbO}_{3}$ [6-8]. Although $\mathrm{LiNbO}_{3}$ possesses a strong electro-optic coefficient allowing efficient tuneable devices, it also suffers from mid-IR absorption, in this case at wavelengths beyond $\lambda=5 \mu \mathrm{m}$. Bentini et al. [8] demonstrated a miniature $\left(60 \mathrm{~mm}^{2}\right)$ FTIR electro-optic spectrometer based on a $\mathrm{C}: \mathrm{LiNbO}_{3}$ material platform. The device was evaluated using a He-Ne laser and a Xe-arc lamp. The maximum achieved OPD was $\sim 0.2 \mathrm{~mm}$ which corresponds to a resolution of $50 \mathrm{~cm}^{-1}$. Another very interesting approach that was first demonstrated by Kita et al. is the on-chip digital Fourier transform spectroscopy (dFT) [11]. In this technique, the spectrometer comprised of a MZI which is further split into multiple cascaded MZIs in each arm. Each of these MZIs has a different OPD achieved through physically unbalanced arms. The total OPD created at the output of the spectrometer is not continuously tuned like in a traditional FTIR spectrometer, rather it is digitised through a combination of binary states of the unbalanced MZI and a set thermo-optic switches which are embedded in the cascaded MZIs. In [11] the authors presented a 64 (6 cascaded MZIs) channel plug and play dFT spectrometer chip that measured a few $\mathrm{cm}^{2}$ owing to the use of SOI technology (large RI contrast). This device was designed and evaluated in the $\mathrm{C}$ band wavelength range $(\sim 1550 \mathrm{~nm})$ and achieved a resolution of $0.2 \mathrm{~nm}$ at the cost of $99 \mathrm{~mW}$. It should be noted that designing such a spectrometer using a low RI contrast waveguide platform would result in a much larger device footprint. During the past decade, $\mathrm{SiGe}$ waveguides have made a strong appearance in mid-IR integrated optics [14, 15], and on-chip spectrometers employing this platform have already been demonstrated in the literature $[16,17]$. These devices show great potential for use in the mid wave infrared region (MWIR), with high achievable spectral resolutions and low waveguide propagation losses [15]. The reported propagation losses of the SiGe waveguides were of the order of 2-3 dB/cm at LWIR [14], whereas losses down to $1 \mathrm{~dB} / \mathrm{cm}$ were recently reported in $\mathrm{Ge}$ on $\mathrm{Si}(\mathrm{GOS})$ waveguides at 11 $\mu \mathrm{m}$ wavelength [18].

In the search for an appropriate waveguide platform suitable for realising a miniature spectrometer operating over most of the mid-IR spectral region, III-V semiconductors offer very attractive characteristics. For example, GaAs has been shown to provide waveguide propagation loss of less than 0.5 $\mathrm{dB} \mathrm{cm} \mathrm{cm}^{-1}$ at $\sim \lambda=1310 \& 1550 \mathrm{~nm}$ [19-21] and is transparent from $0.9 \mu \mathrm{m}$ to $17 \mu \mathrm{m}$, rendering it more suitable for waveguided MIR applications than either $\mathrm{Si}$ or $\mathrm{LiNbO}_{3}$. III-V semiconductors have been intensively exploited for optoelectronic applications due to their outstanding electronic and optical properties [22]. Many common passive and active electronic and optoelectronic devices are based on III-V semiconductors and can be found in devices in everyday use (LEDs, ultra-fast transistors) as well as in devices used in optical communications and other photonic applications (sources, detectors)

$\mathrm{GaAs}$ is widely used in tuneable integrated optoelectronic devices such as switches and modulators [23-25]. These devices utilise the electro-optic effect (EOE) exhibited by the GaAs lattice when subjected to an external electric field. However, GaAs has a relatively low electro-optic coefficient (EOC) of around $1.5 \mathrm{pm} / \mathrm{V}$. The strength of this effect has proven to be enough for use in optical modulators operating in the communication band $(\lambda=1550 \mathrm{~nm})$ where only a $\pi$ radian phase shift is normally needed.

Table 1. Comparison of the optical properties of candidate tuneable waveguide materials $[26,27]$

\begin{tabular}{|l|l|l|l|}
\hline Material & $\begin{array}{l}\text { TOC in the } \\
\text { MIR }\left(\times 10^{-4}\right)\end{array}$ & EOC $(\mathrm{pm} / \mathrm{V})$ & $\begin{array}{l}\text { Transmission } \\
\text { band }(\mu \mathrm{m})\end{array}$ \\
\hline $\mathrm{LiNbO}_{3}$ & 0.289 & 30 & $0.35-5$ \\
$\mathrm{Si}$ & 1.59 & - & $1.1-6.5$ \\
$\mathrm{GaAs}$ & 2 & $\sim 1.5$ & $0.9-17.3$ \\
$\mathrm{In}_{0.49} \mathrm{Ga}_{0.51} \mathrm{P}$ & $\begin{array}{l}2(@ 1550 \\
\mathrm{nm})\end{array}$ & - & $0.65-24$ \\
\hline
\end{tabular}

However, for a Fourier Transform spectrometer where $>100$ $\pi$ radians phase shift is required, more significant refractive index tuning is needed and the electric fields required are not practical and could also damage the device. In contrast to its low EOC, GaAs possesses a rather high thermo-optic coefficient (TOC) of approx. $2 \times 10^{-4} \mathrm{~K}^{-1}$. Edilson et al. [28] have demonstrated an ultra-compact thermo-optic switch based on a GaAs platform using a photonic crystal waveguiding structure operating at $\lambda=1550 \mathrm{~nm}$. Their device performed well despite the need for high a temperature excursion due to the small active arm length used, making it a promising platform for efficient optical tuning. EOCs, TOCs and transmission windows for candidate materials are given in table 1.

In this work, we describe the design and simulation of a thermo-optically tuned spectrometer based on a GaAs on $\mathrm{In}_{0.49} \mathrm{Ga}_{0.51} \mathrm{P}$ material platform. The device utilises an integrated tuneable Mach Zehnder Interferometer (MZI) with a metallic heater. The optical and thermal properties of the 
structure were modelled using Lumerical and COMSOL Multiphysics, respectively. The optimised layer structure dimensions and the thermal/power performance of the device are presented and the spectrometer performance predicted.

\section{Waveguide material system and spectrometer configuration}

The layer structure of the proposed waveguide platform is shown in figure 1. A commonly-used cladding for GaAs waveguide devices [23-25, 28] is $\mathrm{Al}_{\mathrm{x}} \mathrm{Ga}_{1-\mathrm{x}} \mathrm{As}$, because $\mathrm{Al}_{\mathrm{x}} \mathrm{Ga}_{1-}$ ${ }_{x}$ As is lattice-matched to $\mathrm{GaAs}$ for any composition. However, $\mathrm{In}_{0.49} \mathrm{Ga}_{0.51} \mathrm{P}$ is a promising alternative cladding material, since it suffers less from oxidation than $\mathrm{Al}_{\mathrm{x}} \mathrm{Ga}_{1-\mathrm{x}} \mathrm{As}$ and offers good quality and uniform epitaxial layers [27, 29] with a lattice mismatch to GaAs as low as $\left(\Delta \alpha / \alpha=10^{-4}\right)$ [30]. $\operatorname{In}_{0.49} \mathrm{Ga}_{0.51} \mathrm{P}$

also offers low dislocation propagation velocity and strong etch selectivity [27]. $\mathrm{In}_{0.49} \mathrm{Ga}_{0.51} \mathrm{P}$ has a wider low-absorption transmission window in the mid-IR than GaAs, spanning from $\sim 0.65 \mu \mathrm{m}$ to $\sim 24 \mu \mathrm{m}$ as shown in [27], so it will not limit the spectral transmission range of the waveguides. For these reasons, $\mathrm{In}_{0.49} \mathrm{Ga}_{0.51} \mathrm{P}$ was selected as the cladding material. The refractive index (RI) data for GaAs were extracted from the Sellmeier coefficients provided in [31], and for $\mathrm{In}_{0.49} \mathrm{Ga}_{0.51} \mathrm{P}$, the RI data were deduced from Sellmeier coefficients given in [27]. Table 2 contains the optical constants used in the simulations; the optical constants for the $\mathrm{Ni}$ heater were used directly from Lumerical's database. In the first instance, as proof of principle, the spectrometer was designed to operate between $\lambda=3 \mu \mathrm{m}$ and $4 \mu \mathrm{m}$ using the theoretical principles outlined in section 3 . This wavelength range is convenient for initial fabrication and characterisation and will allow validation of the design procedure before realisation of a long-wavelength spectrometer for operation between $\lambda=8$ and $12 \mu \mathrm{m}$.

The complete system will comprise an input optical signal whose spectrum is to be determined, a Mach-Zehnder Interferometer with long spiral arms to allow a sufficient phase shift excursion to be realised, Ni heater electrodes matching the spiral waveguides for thermo-optic tuning, and a photodetector (on-chip or off-chip), as shown in figure 2. A key section to be optimised is the thermal tuning region, which will yield the change in modal effective index with electrode current and hence define the MZI arm length required to achieve the necessary phase excursion. The waveguide cladding is not shown in figure 2, for clarity, but the full waveguide layer structure in the tuning section is shown in cross-section in figure $4(\mathrm{f})$.

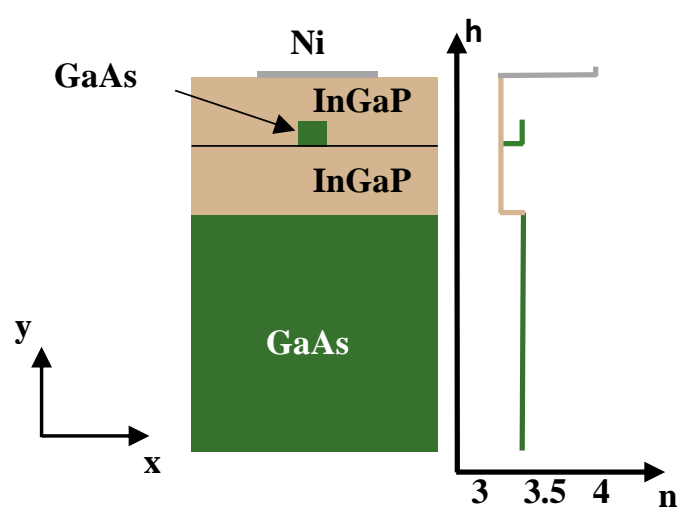

Figure 1. GaAs/InGaP layer structure and refractive index profile

Table 2. Empirical Sellmeier Coefficients for GaAs and $\mathrm{In}_{0.49} \mathrm{Ga}_{0.51} \mathrm{P}$ $[27,31]$

\begin{tabular}{|c|c|}
\hline Material & $\mathrm{GaAs} / \mathrm{In}_{0.49} \mathrm{Ga}_{0.51} \mathrm{P}$ \\
\hline $\mathrm{A}$ & $8.950 / 6.058$ \\
$\mathrm{~B}$ & $2.054 / 3.270$ \\
$\mathrm{C}(\mu \mathrm{m})$ & $0.6245 / 0.459$ \\
\hline
\end{tabular}

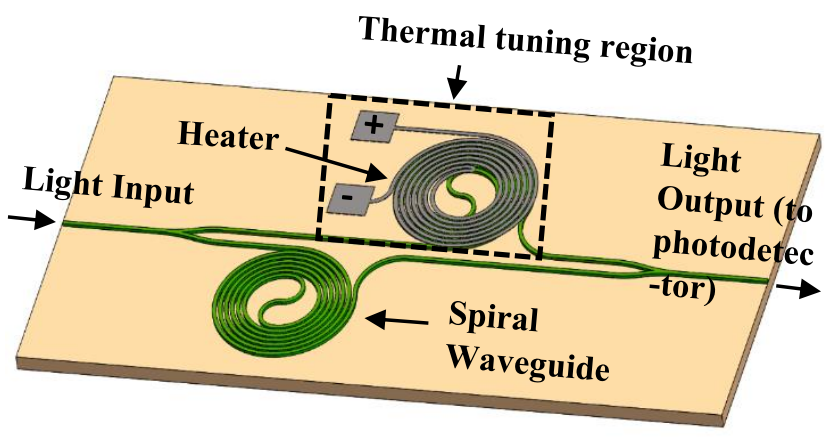

Figure 2. Spectrometer Layout

\section{Spectrometer thermal scanning}

In this section the thermal requirements to achieve the spectral resolution required are discussed, leading to definition of the MZI path length and allowing full optical and thermal design of the spectrometer chip. A resolution of 10 wavenumbers (10 $\mathrm{cm}^{-1}$ or $0.3 \mathrm{THz}$ ) was selected as this is sufficient for analysis of liquid samples such as those found in biomedical diagnostics or water pollution sensing. The wavenumber resolution, $\Delta \mathrm{s}$, is determined by the length over which the optical path-length difference in the interferometer arms is scanned, the OPD, according to (Eq. (1)):

$$
\Delta s=\frac{1}{O P D}
$$


so that in a thermo-optically tuned spectrometer the resolution is given by:

$$
\Delta s=\frac{1}{L \frac{d n}{d T} \Delta T}
$$

where $\mathrm{L}$ is the active length of the tuneable structure, $\left(\frac{d n}{d T}\right)$ is the TOC and $\Delta \mathrm{T}$ is the temperature excursion scanned. The active length is defined as the section of the waveguide that is subjected to heating. In order to limit the power consumption of the device, the maximum heater temperature was chosen to be less than $100 \mathrm{~K}$.

The TOC of $\operatorname{In}_{0.49} \mathrm{Ga}_{0.51} \mathrm{P}$ was taken from [32] and the TOC of GaAs was taken from [26]; both are approximately $2 \times 10^{-4}$ $\mathrm{K}^{-1}$. The wavelength dispersion and temperature dependence of $\left(\frac{d n}{d T}\right)$ are important factors to consider, as the chip will operate over a wide range of wavelengths and temperatures during scanning. The TOC of GaAs is given for three different wavelengths $(1.15 \mu \mathrm{m}, 3.39 \mu \mathrm{m}$ and $10.6 \mu \mathrm{m})$ in [26]. For wavelengths of $3.39 \mu \mathrm{m}$ and $10 \mu \mathrm{m}$ the value of $\left(\frac{d n}{d T}\right)$ was the same, so it was assumed that $\left(\frac{d n}{d T}\right)$ does not vary over the wavelength range of interest. Della Corte et al. [33] have studied the temperature dependence of the thermo-optic coefficient of semiconductors including GaAs. They presented empirical equations based on polynomial fits of real data and found that at $\lambda=1523 \mathrm{~nm}$, the GaAs TOC only shows a small change of the order of $\sim 0.02 \times 10^{-4} \mathrm{~K}^{-1}$ between 300 and $400 \mathrm{~K}$. Since we have chosen to limit the temperature excursion to $<100 \mathrm{~K}$ in our device, the temperature dependence of the TOC was neglected in this study. TOC wavelength dispersion and temperature dependence data for $\operatorname{In}_{0.49} \mathrm{Ga}_{0.51} \mathrm{P}$ were not available in the literature and thus were neglected.

From Eq. (2), for a given material system, the spectral resolution can be reduced either by increasing $\mathrm{L}$ or by increasing $\Delta \mathrm{T}$. With $\Delta \mathrm{T} \sim 100 \mathrm{~K}$ and assuming a modal TOC of $\sim 2 \times 10^{-4} \mathrm{~K}^{-1}$, the active length required to achieve a resolution of $10 \mathrm{~cm}^{-1}$ will be $\sim 50 \mathrm{~mm}$. This may be achieved in a small device footprint by using spiral waveguide arms as shown in figure $2[9,34]$. To allow for design and fabrication tolerances, and to limit the temperature excursion below 100 $\mathrm{K}$, an active length $L$ of $60 \mathrm{~mm}$ is chosen for detailed simulations to refine the design. The key design parameters are summarised in table 3 below.

Table 3. Mid-IR Spectrometer Scanning Region Design Parameters

\begin{tabular}{|l|l|}
\hline Desired Spectral Resolution & $10 \mathrm{~cm}^{-1}$ \\
OPD required & $1 \mathrm{~mm}$ \\
MZI arm active length chosen & $60 \mathrm{~mm}$ \\
Estimated modal TOC & $2 \times 10^{-4}$ \\
Total $\Delta \mathrm{T}$ required for $10 \mathrm{~cm}^{-1}$ resolution & $83.33 \mathrm{~K}$ \\
\hline
\end{tabular}

The performance of a thermally-tuneable optical device is strongly dependent on the thermal properties of the materials comprising the structure. Our waveguide platform is based on III-V semiconductors which are characterised by low thermal conductivities $(k)$. Depending on a device's specifications, either low or high $k$ may be required. For example, for modulators, rapid switching speeds are essential, which can be achieved with the help of high $\mathrm{k}$ materials. However, that comes at the cost of higher power dissipation [22]. In the case of a spectrometer, ultra-fast measurement times are not needed, thus the power consumption of the device is mitigated by using materials with low $k$.

\section{Modelling Results}

With the required physical path length and OPD defined, (i) the waveguide structure and layout and (ii) the electrode/materials configuration must be simulated to finalise the detailed design. This is to ensure (i) low waveguide and spiral loss and (ii) realisation of $\Delta \mathrm{T}<100 \mathrm{~K}$ at the waveguide core in the active arm, with low crosstalk $(\Delta \mathrm{T})$ in the "reference" arm.

\subsection{Waveguide structure modelling and optimisation}

The first waveguide design consideration was to ensure monomode operation of the waveguides throughout the spectral region of interest, between $\lambda=3 \mu \mathrm{m}$ and $4 \mu \mathrm{m}$, as the presence of higher order modes would yield spurious interferences [35].

For the calculation of the mode effective refractive indices $\left(\mathrm{N}_{\mathrm{eff}}\right)$ and propagation losses due to leakage to the substrate, absorption by the electrode, and bends, the 2D Finite Difference Eigenmode (FDE) solver in Lumerical MODE was used. Perfectly Matched Layers (PML) and metal boundaries were applied where appropriate. The estimation of the propagation loss was extracted from the extinction coefficients $(\mathrm{k})$ of the modes. To calculate the bend loss, the waveguides were modelled using the simulated bend option which is included in the FDE solver.

\subsubsection{Single mode optimization}

Figure 3(a) and (b) presents the $\mathrm{N}_{\text {eff }}$ of the fundamental and first order TE and TM modes for different waveguide widths at $\lambda=3 \mu \mathrm{m}$ where the core index is $\sim 3.33$ and the cladding index is $\sim 3.067$. Figure 3(c) shows the fundamental TE and TM modes for different waveguide widths at $\lambda=4 \mu \mathrm{m}$ where the core and cladding indices are $\sim 3.325$ and $\sim 3.061$ respectively. Simulating the waveguide for monomode operation at the shortest desired wavelength ensures monomode operation for longer wavelengths as well, as the $\mathrm{N}_{\text {eff }}$ of modes reduces with wavelength. After performing a wide-ranging waveguide dimension optimisation simulation varying both the waveguide thickness and width, a thickness of $1.5 \mu \mathrm{m}$ was selected since it was the smallest to provide good fundamental mode confinement while the higher order modes are cut off. Choosing a smaller waveguide thickness aids waveguide fabrication since it is more convenient to fabricate wider than thicker waveguides. The black line with circular points and the pink line with triangular points 
represent the $\mathrm{N}_{\text {eff }}$ of the fundamental/first order TE and TM modes, respectively. The blue dashed line in figure 3(b) is the mode cut-off limit for Mode 1 (the cladding refractive index). The higher-order mode is cut off for a waveguide width of 1.6 $\mu \mathrm{m}$ or less for both the TE and TM polarisation. The difference between the effective indices for the two polarisations is small due to the relatively low index contrast between the waveguide core and cladding $(\Delta \mathrm{n} \approx 0.26)$ compared to other platforms such as SOI.
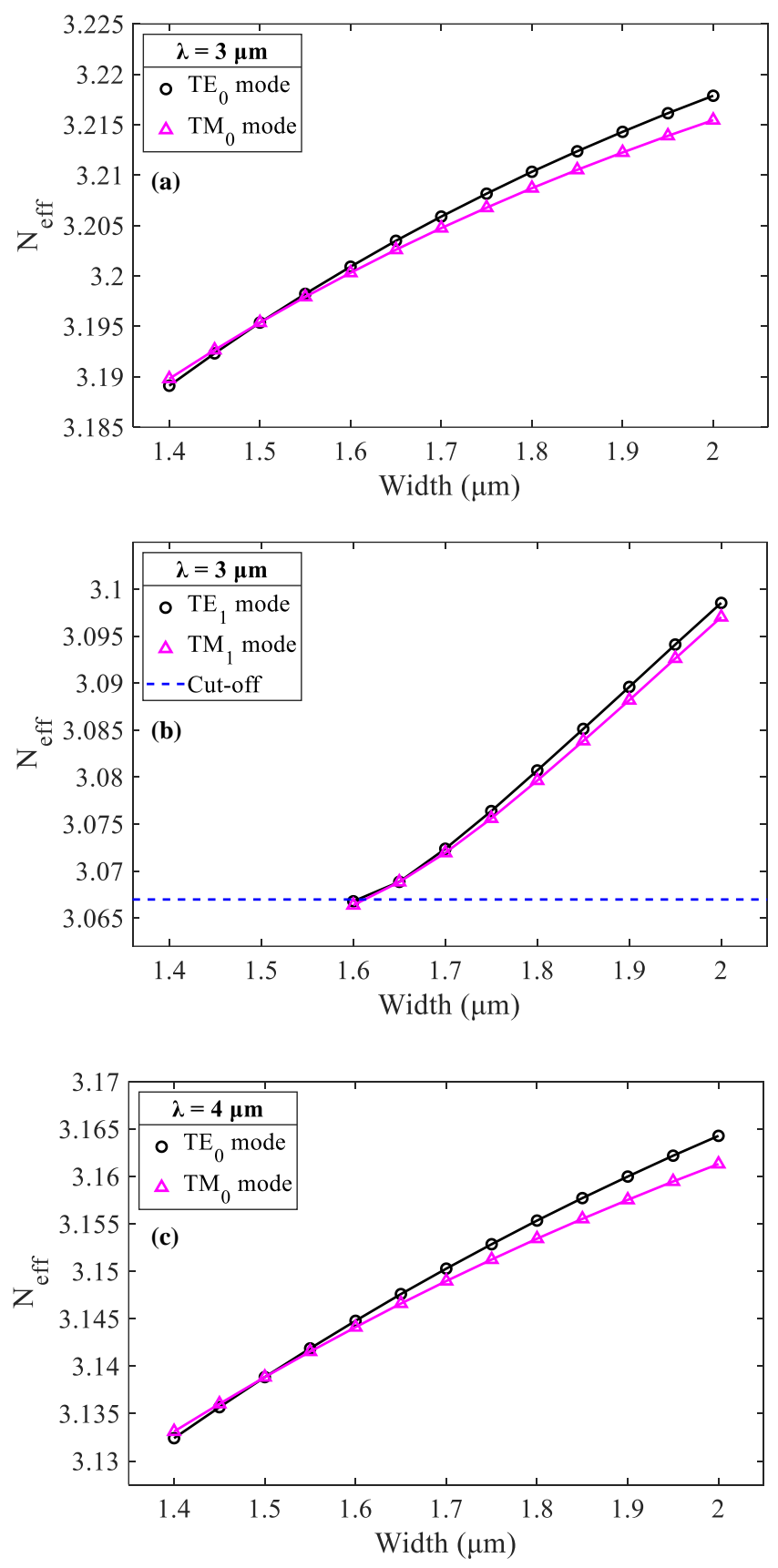

Figure 3. Modal $\mathrm{N}_{\text {eff }}$ vs waveguide width for (a) fundamental TE and TM modes at $\lambda=3 \mu \mathrm{m}$ (b) $1 \mathrm{st}$ higher order TE and TM modes at $\lambda=3 \mu \mathrm{m}$ (including the mode cut-off effective index) and (c) fundamental $\mathrm{TE}$ and TM modes at $\lambda=4 \mu \mathrm{m}$.
A waveguide width of $1.6 \mu \mathrm{m}$ was chosen to ensure monomode operation and maximum mode confinement in both polarisations over the operating range from $\lambda=3 \mu \mathrm{m}$ to 4 $\mu \mathrm{m}$. The 1st higher order mode was cut-off for all widths at $\lambda=4 \mu \mathrm{m}$ and thus was not included in the graphs.

\subsubsection{Cladding thickness optimization}

Once the waveguide core dimensions were established, the over and under cladding thicknesses were modelled and optimised to limit losses by metal electrode absorption and radiation into the substrate. The wavelength was set at $4 \mu \mathrm{m}$ in order to model the situation with the highest expected loss, which will occur when the mode cross-section is largest at the longest wavelength. The cladding layers must provide enough optical isolation from the highly absorptive metallic heater and the high index substrate while still being thin enough to be easily grown and, in the case of the over cladding, to allow effective heat transfer. The optimum cladding thickness is therefore the smallest which gives an acceptable loss.

Figure 4(a) and (b) show the simulated loss vs. over cladding thickness, for under cladding thicknesses ranging from $3 \mu \mathrm{m}$ to $7 \mu \mathrm{m}$, for both polarisations. An acceptable loss of $<0.06 \mathrm{~dB} \mathrm{~cm}^{-1}$ is obtained for a $5 \mu \mathrm{m}$ layer thickness for both the over and the under cladding for TE polarised light. It should be noted that, due to the core thickness of $1.5 \mu \mathrm{m}$, the actual distance between the heater and the top surface of the waveguide core is $3.5 \mu \mathrm{m}$ as can be seen in figure 4(f). The results obtained for TM polarised light, in figure 4(b), show that the losses are significantly higher than for the TE polarisation ( $\sim 5.7 \mathrm{~dB} \mathrm{~cm}^{-1}$ for the same layer thickness). This is due to the nature of the TM polarization and its overlap/interaction with the metal layer and high index substrate.

In figure 4(c) and (d), the electric field distributions for the TE and TM modes, are given for the $\mathrm{x}$ and $\mathrm{y}$-direction, respectively, for $5 \mu \mathrm{m}$ over and under cladding thicknesses. In the $\mathrm{x}$-direction the E-field distribution for both polarisations is very similar and shows the expected modal distribution for a dielectric waveguide. However, in the y-direction the TE Efield amplitude drops to zero where the field is in contact with the heater due to the metallic nature of $\mathrm{Ni}$ (high extinction coefficient). The TM E-field shows a large increase in amplitude at the interface of the cladding with the metallic heater. This E-field amplitude spike observed at the dielectric/metal interface stems from the excitation of surface plasmon polaritons at the metal surface [36], and leads to strong absorption of the TM mode. A thicker under cladding could be chosen to further increase optical isolation between the propagating mode and the high refractive index substrate, however, it is more challenging to fabricate high quality layers of larger thickness, and the substrate can be used as high order mode stripper [37]. Polarised quantum cascade sources can be used to ensure operation in the TE polarisation. 

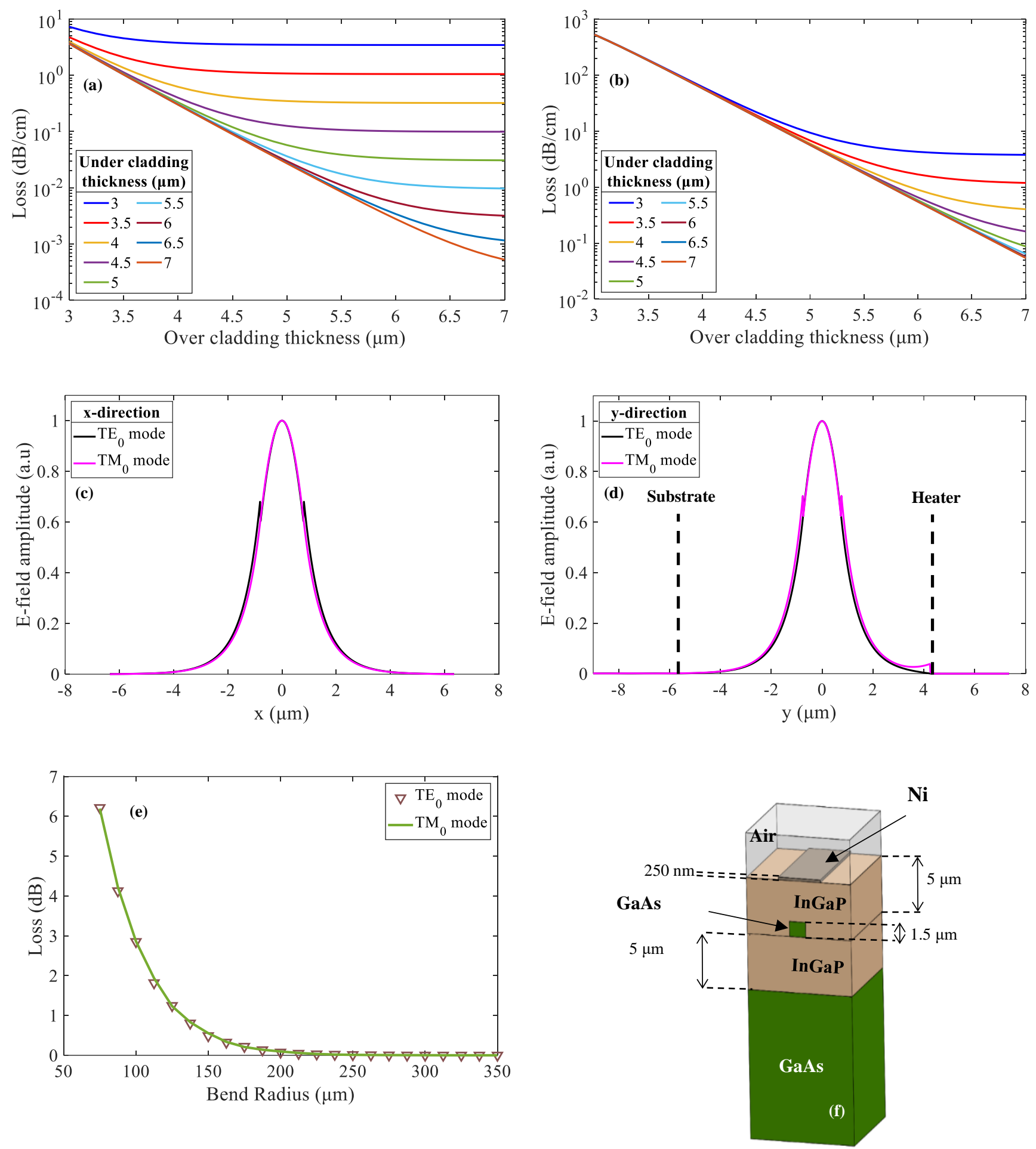

Figure 4. Heater/substrate loss vs cladding thickness for (a) TE mode and (b) TM mode. (c) TE\&TM mode profile line graph (xdirection), (d) TE\&TM mode profile line graph (y-direction). The mode profiles were plotted using a thickness of $5 \mu \mathrm{m}$ for both over and under claddings (e) Loss per $180^{\circ}$ bend and (f) Optimised Layer structure 


\subsubsection{Curved waveguide optimisation}

With the basic waveguide design established, the bend losses of the waveguide platform were estimated. As can be seen in figure 2, the spiral arms of our design contain two small-radius bends in the middle of the spiral. These bends can introduce high radiation losses if not designed properly. For that reason, the radiation bend losses for the specified waveguide core dimensions were estimated at $\lambda=4 \mu \mathrm{m}$. The loss per $180^{\circ}$ bend is plotted in figure 4(e) for both TE and TM polarisation. An acceptable loss per bend of $<0.1 \mathrm{~dB}$ is observed for $\mathrm{R} \geq 200$ $\mu \mathrm{m}$ and the loss behaviour is very similar for both polarisations. The modelled part of the layer structure used in these simulations consisted of only the core and the cladding layers, thus it only illustrates the bend-induced loss alone and not the loss due to the metal heater and the substrate, which was modelled and estimated separately as above. The effect of the presence of the metal heater and the substrate on the bend loss will practically be negligible because of the thick enough cladding $(5 \mu \mathrm{m})$. A conservative minimum bend radius of 350 $\mu \mathrm{m}$ was chosen for the final design to ensure low bend losses. The design of the spiral thermal scanning waveguide section is shown in figure 5 . The spiralled waveguides are spaced by $50 \mu \mathrm{m}$ and the $60 \mathrm{~mm}$ length of waveguide is contained in a < $2.5 \mathrm{~mm}$ diameter footprint. The large spiral inter-turn spacing was selected such that it eliminates any coupling between the waveguides. An FDE simulation showed that for a spacing of $\sim 10 \mu \mathrm{m}$ and above, insignificant coupling takes place between two straight waveguides at $\lambda=4 \mu \mathrm{m}$. However, considering the mode shift inside the bent waveguides, this spacing needs to be larger and thus a conservative spacing of $50 \mu \mathrm{m}$ was selected.

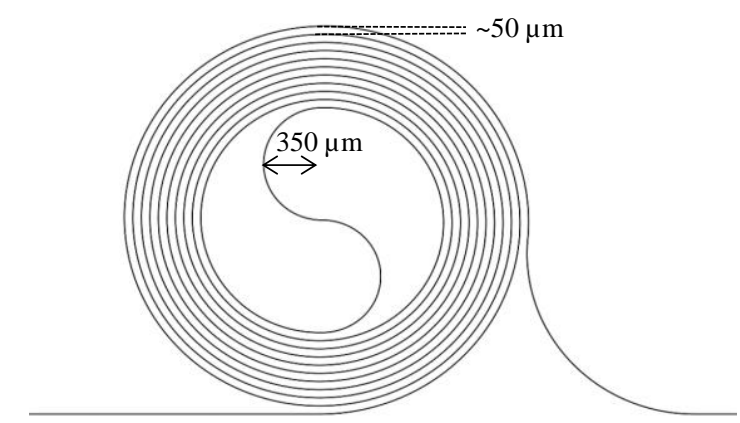

Figure 5. Final design of the spiral waveguide section

\subsection{Thermal Modelling}

Having established the optical design of the spectrometer, thermal modelling was carried out to (i) determine the current required to achieve a temperature elevation of $\sim 83 \mathrm{~K}$ over the core region of the waveguides, (ii) estimate the electrical current/power consumption during thermal scanning when the device is placed on a heat sink or thermally insulated platform (iii) consider the impact of thermal crosstalk with the MZI reference arm and (iv) determine the response speed to thermal scanning.

\subsubsection{Thermal simulation layout and procedure}

In order to estimate the power and time needed for sufficient thermal tuning, transient thermal analysis of the layer structure was performed. One way to mitigate the total power dissipation is to reduce the active area of the heater. Considering that the spiral arms of the MZI contain regions that are not occupied by large lengths of waveguides (centre), we propose a heater geometry similar to that of the spiral waveguides as shown in figure 2 so that the heat produced by the heating element is not wasted on areas where there are no active waveguides. The simulated geometry is shown in Fig 6 . The thickness and width of the Ni heater were chosen to be $250 \mathrm{~nm}$ and $25 \mu \mathrm{m}$, respectively. The dimensions of the heater determine its total resistance, so that the current required to achieve a specified temperature excursion is determined by the dimensions of the heater. Moreover, heating elements are sensitive to high currents and can suffer catastrophic failure during operation, if not designed properly. One effect that is common in micro and nano sized devices, is that of electromigration. Preliminary calculations predicted that this heater will not experience any significant electromigration for the required currents. Micro heaters with similar thicknesses to our design have been demonstrated in the literature [38, 39]. The over-cladding thickness was set to $5 \mu \mathrm{m}$, in accordance with the optical design in section 4.1.2, and under-cladding thicknesses of $5 \mu \mathrm{m}, 15 \mu \mathrm{m}$ and $25 \mu \mathrm{m}$ were modelled, to study the effect on the performance due to the low thermal conductivity of the cladding material. The substrate thickness was $350 \mu \mathrm{m}$. A 3D model representation of the heater geometry was created in Solidworks and then imported to COMSOL where it was analysed using the Electromagnetic Heating physics module. The heat transport equation [22] was solved in COMSOL for a mixture of triangular and quadrilateral meshes. In order to avoid any conflicts when creating the meshes and minimise the computational expense due to the high aspect ratio geometries, the single layer shell interface was utilised in this study and the each of the two contact pads shown in figure 6 , were used as the positive and ground terminal, respectively. The ambient temperature was set to the default $(293.15 \mathrm{~K})$ and the boundary conditions were set up similar to [40] as follows: Free air convection (5 $\mathrm{W} / \mathrm{m}^{2} \mathrm{~K}$ ) on all surfaces that are exposed to air and an open boundary on all the sides of the geometry. The bottom surface of the substrate was modelled both as a heat sink (293.15 K) and as thermally insulated. These two different conditions for the bottom boundary were studied as both are feasible in real device operation and have a significant impact on the performance.

Radiation losses were neglected since the studies were limited to temperature increases below $200 \mathrm{~K}$ using a temperature stop condition. The power dissipation of the heater element was obtained by integration of the heater's surface loss density. However, it can also be estimated using the total resistance of the $\mathrm{Ni}$ heater and the input current/voltage. 


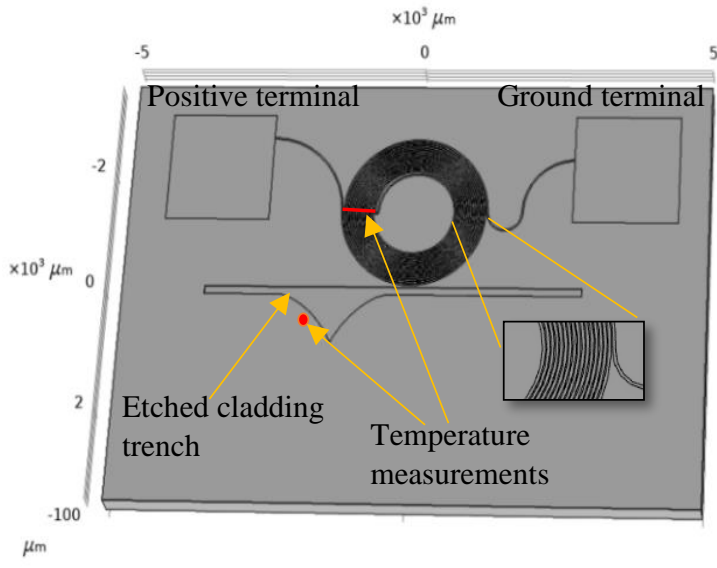

Figure 6. Geometry modelled in COMSOL

The temperature dependence of the thermal conductivity and specific heat was taken into account for all the materials involved. The values for the thermal conductivity $(\mathrm{k})$, heat capacity (c) and density ( $\rho$ ) of the materials used in these simulations were extracted from [41] and from COMSOL's library and are summarised in table 4.

Table 4. Physical parameters of the materials at room temperature $[41 \&$ COMSOL]

\begin{tabular}{|llll|}
\hline Material & \multicolumn{1}{c}{$k$} & \multicolumn{1}{c}{$c$} \\
$(\mathrm{~W} / \mathrm{K} \mathrm{m})$ & $(\mathrm{J} / \mathrm{K} \mathrm{kg})$ & $\begin{array}{c}\rho \\
\left(\mathrm{g} / \mathrm{cm}^{3}\right)\end{array}$ \\
\hline $\mathrm{GaAs}$ & 46 & 322 & 5.320 \\
$\mathrm{In}_{0.49} \mathrm{Ga}_{0.51} \mathrm{P}$ & 5.2 & 465.59 & 4.46728 \\
Nickel & 91.45 & 441.46 & 8.900 \\
\hline
\end{tabular}

The heater temperature was recorded using the line average temperature over the line marked "temperature measurement" in figure 6.

Figure 6 shows a trench, etched to the bottom of the lower cladding, placed between the MZI arms in order to mitigate heat transfer between them. The second (reference) MZI arm itself is not shown in figure 6 , as it is not directly heated. However, any heating of the reference arm due to heat transfer will reduce spectrometer tuning, as the differential optical path length achieved will be reduced. Therefore, the temperature at the circular point shown in figure 6 , where the second spiral arm is closest to the heater, is monitored to allow worst-case cross-heating analysis. Note that the second spiral arm is placed at a lateral offset of $2.5 \mathrm{~mm}$ (spiral centre-centre), as shown schematically in figure 2 , to further reduce crosstalk.

\subsubsection{Thermal behaviour of the tuned MZI arm}

The temperature in the tuned waveguide arm is plotted as a function of time in figure 7 , with a $5 \mu \mathrm{m}$ thick under-cladding for four different heater currents, for (a) a heat-sink substrate boundary and (b) an insulated substrate boundary. In all cases, the transient response of the thermal tuning is in the order of $\mathrm{ms}$, which is a sufficient rate for many spectral measurements, and the temperature increase is greater for higher currents as expected. In the case of good heat-sinking, which maintains room temperature at the bottom of the substrate (figure 7(a)), the temperature rises and saturates within $20 \mathrm{~ms}$ for each input current, due to the steady heat dissipation through the heat sink. In the case of a perfectly thermally insulated substrate (figure 7 (b)) the heat is not dissipated through the substrate and thus keeps rising with time even at lower input currents. The temperature of each configuration was also recorded at longer times (up to $250 \mathrm{~ms}$ ). It was confirmed that temperature saturation continues in the heat sink case, while the temperature continued to increase in the thermally-insulated case at heating times up to $250 \mathrm{~ms}$. It should be noted that several assumptions were made in order to simplify the modelling of the device, including the perfect insulation/heat sinking of the bottom surface and the open side boundaries. In reality, there will always be imperfect heat dissipation through the bottom surface and convective heat transfer from all the exposed sides of the device.
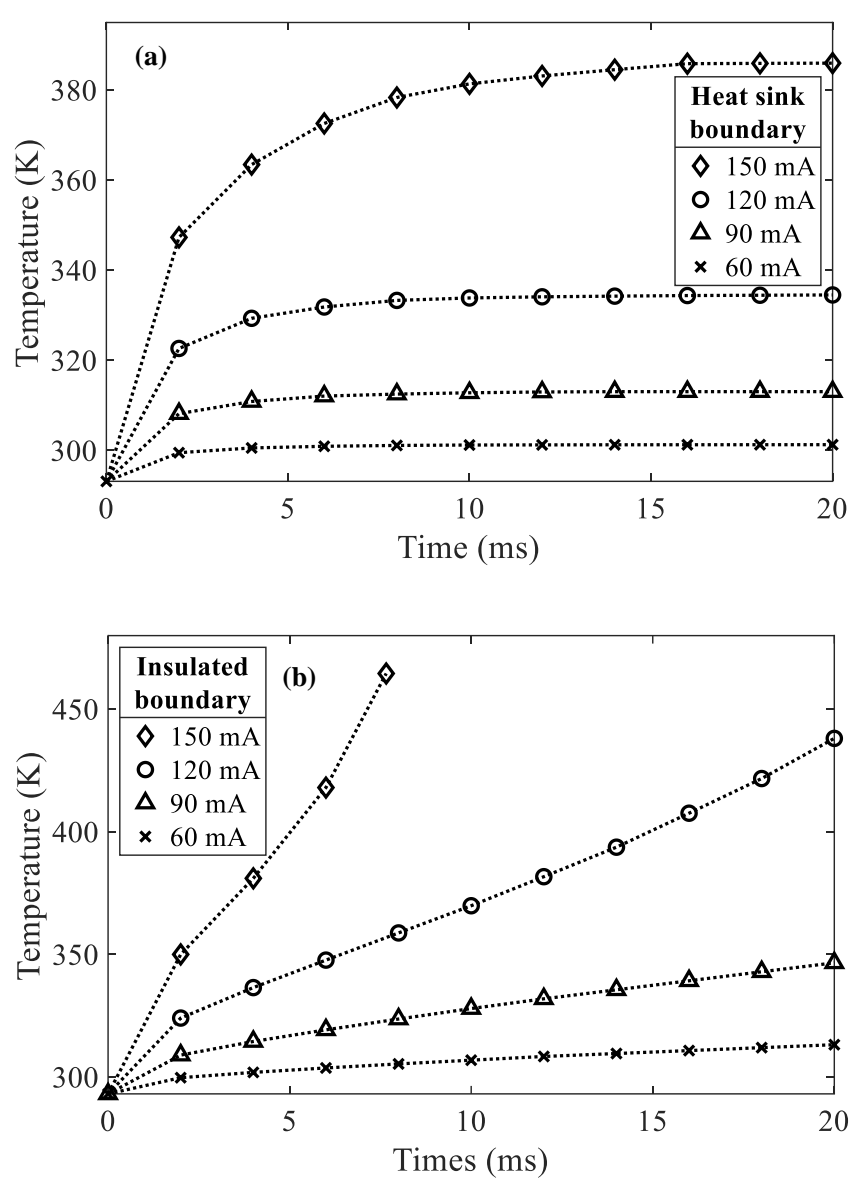

Figure 7. Tuneable waveguide arm temperature rise with time for several drive currents: (a) bottom surface set as a heat sink and (b) thermally insulated bottom surface 


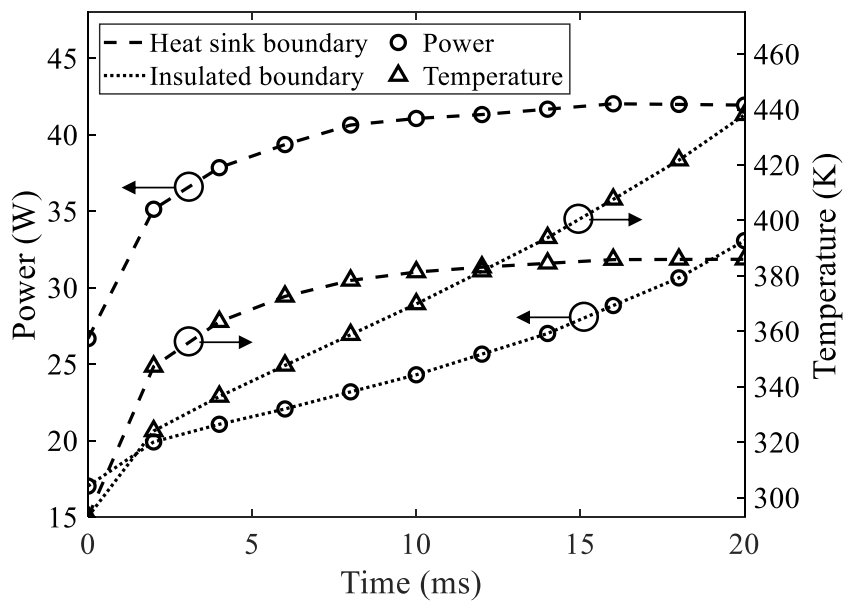

Figure 8. Scanning arm temperature and dissipated power vs time for heat-sink and insulating boundary

Dissipated power vs time is shown in figure 8, along with the temperature, for the heat-sink and insulating boundary cases, to illustrate the effect on power consumption. The current has been chosen so that in each case the temperature rise reaches a value close to the required $\sim 83.3 \mathrm{~K}$, in this case $\sim 88.5 \mathrm{~K}$, after $10-12 \mathrm{~ms}$. The power consumption at $10 \mathrm{~ms}$ in the case of ideal heat-sinking is $\sim 41 \mathrm{~W}$, while for ideal thermal insulation and after $12 \mathrm{~ms}$ it is $\sim 25.7 \mathrm{~W}$, resulting in sufficient thermal excursion with lower power consumption. However, the impact of thermal crosstalk and under-cladding thickness must be studied before finalising operating parameters.

\subsubsection{Thermal crosstalk with the reference MZI arm}

A change in the temperature of the MZI reference arm due to heating the scanning arm will reduce the tuning efficiency of the spectrometer by limiting the optical path length difference achieved and thus the spectral resolution. The temperature rise on the reference arm of the spectrometer at the closest point to the heater (crosstalk temperature) was also recorded, giving a worst-case estimate of the crosstalk. As can be seen in figure 9(a), for the heat sink case, the increase in the crosstalk temperature, for all input currents, is very small and thus would have an insignificant effect on the performance of the device. However, if the bottom surface of the substrate is insulated, then heat spreads more significantly across the insulating trench and to the reference arm as shown in figure 9(b). Nonetheless, the temperature elevation is still small and can be compensated with slightly higher current or scan time.
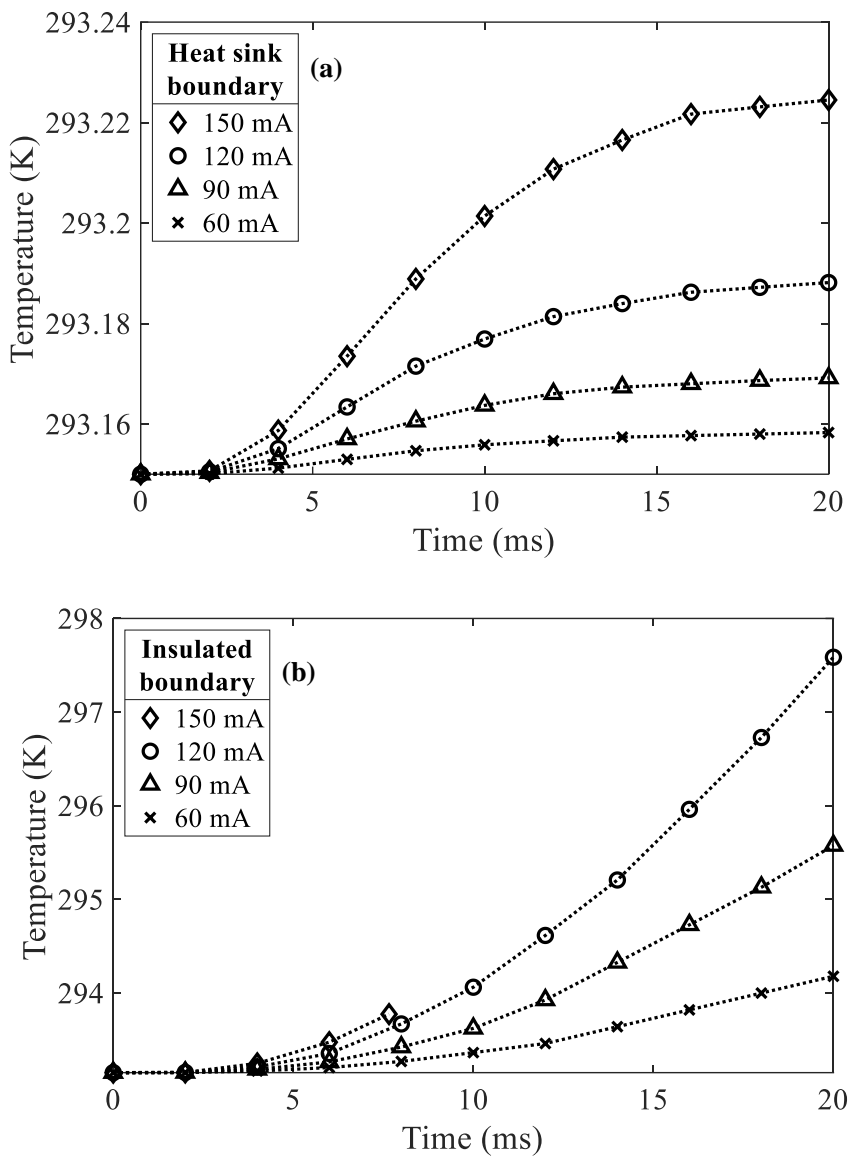

Figure 9. Crosstalk temperature rise with time for (a) bottom surface set as a heat sink and (b) thermally insulated bottom surface

\subsubsection{Under-cladding thickness}

The thermal conductivity of the InGaP cladding material is much lower than that of the GaAs substrate (as seen in table 4), so that a thicker under-cladding will result in reduced power consumption. The waveguide and cross-talk temperatures for under-cladding thicknesses of 5, 15 and 25 $\mu \mathrm{m}$ at $120 \mathrm{~mA}$ input current are given in figure 10 . It can be seen that having a thicker under-cladding would significantly improve the efficiency of the spectrometer by reducing the input current requirement to reach a given temperature. This is due to the reduction of heat dissipation through the thicker cladding to the heat sink and better confinement of heat close to the waveguide region. 

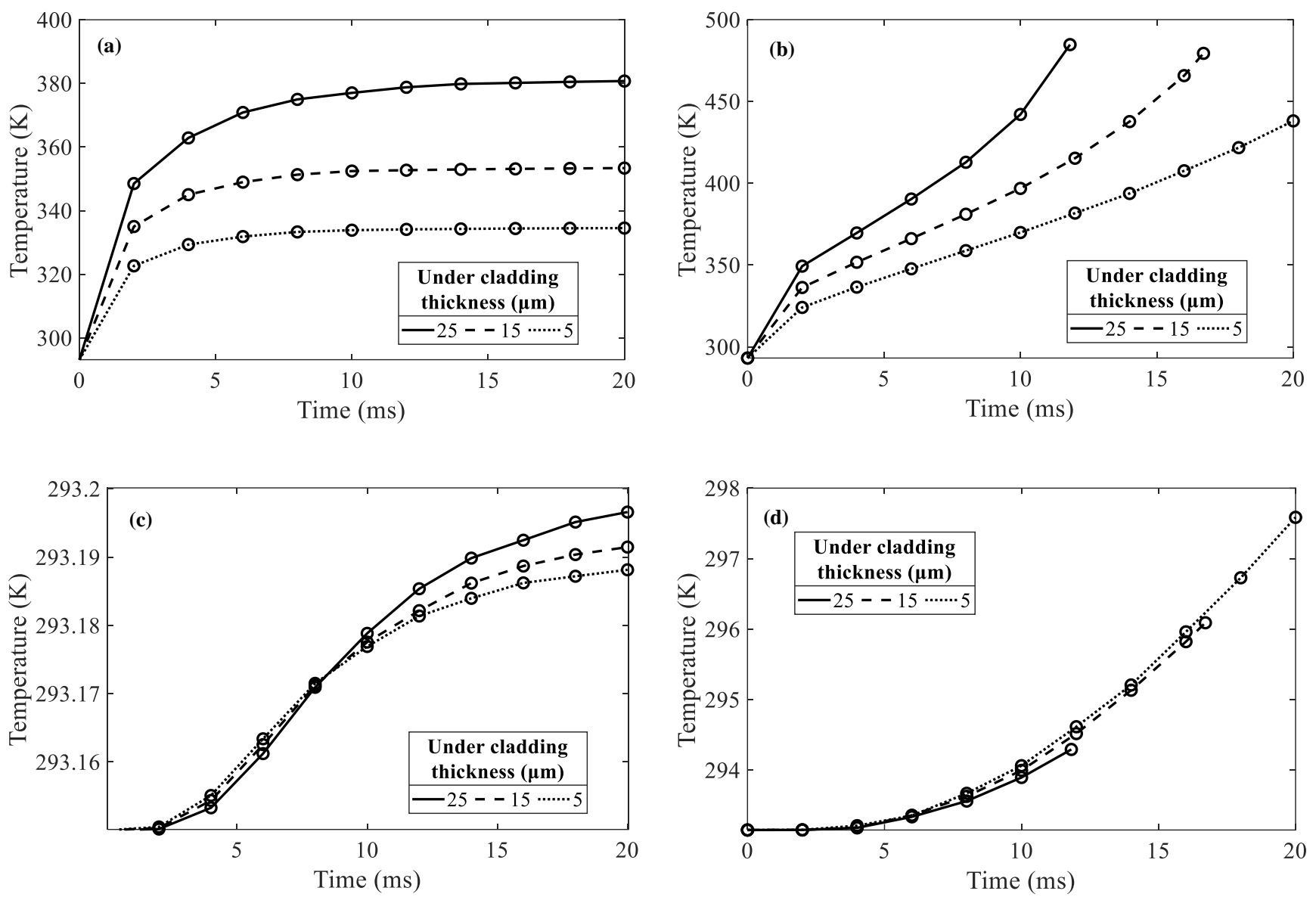

Figure 10. Tuneable waveguide temperature: (a) bottom surface set as a heat sink and (b) thermally insulated bottom surface and crosstalk temperature: (c) bottom surface set as a heat sink and (d) thermally insulated bottom surface for 3 different undercladded thicknesses $(5,15$ and $25 \mu \mathrm{m})$ at $120 \mathrm{~mA}$ input current

\subsubsection{Discussion of predicted thermal tuning performance}

The estimated scanning response time of the order of $10 \mathrm{~ms}$, while not optimum for most modulators, is ample for most spectral measurement systems. A full spectral scan cycle, including the cooling response time of the device, is estimated to take less than $25 \mathrm{~ms}$ in total. During the cooling cycle, the temperature of the tuned waveguides drops to $1 / \mathrm{e}^{2}$ of the total excursion in less than $200 \mu$ s regardless of the device's thermal dissipation design. It should be noted that in the case of insulated bottom surface and in contrast to the heat sink case, the apparent equilibrium temperature is approximately at $1.65 \mathrm{~K}$ above room temperature. The equilibrium temperature is reached at about $10 \mathrm{~ms}$ in both cases. The maximum expected power requirement per scan was found to be as low as $\sim 25.7 \mathrm{~W}$ when the device is properly insulated and $\sim 41 \mathrm{~W}$ when a heat sink is used. However, that reduction in power comes at the cost of higher cross-talk than when the device sits on a heat sink. Nonetheless, the effect of cross-talk when the device is insulated is insignificant given the considerable reduction in power needs. The cross-talk can negatively affect the performance of the device, by limiting the obtainable OPD of the tuneable MZI. However, it is worth noting that in the case of an insulated device, a negative cross-talk time derivative is observed. Thus, it is possible to further improve the efficiency of the device, with prolonged heating (tuning) as well as appropriate post-processing of the obtained data. It should be noted that for both boundary conditions, the average power consumption is significantly lower than the maximum power consumption.

The power requirement of this device is a few times higher than that reported in [9], for example, where a similar resolution was achieved while dissipating only $5.1 \mathrm{~W}$. The reason behind the higher efficiency lies in the use of a $\mathrm{SiO}_{2}$ cladding, which is a poor conductor of heat, reducing unnecessary heat dissipation. In addition, due to the large refractive index contrast of the waveguide and lower operational wavelength $(\sim 1550 \mathrm{~nm})$, very thin claddings and very tight bends were possible. Therefore, the heater could be placed much closer to the waveguide and the device footprint was significantly reduced to the order of $1 \mathrm{~mm}^{2}$. MontesinosBallester et al. [17] have demonstrated a tuneable spatial heterodyne spectrometer on chip based on a SiGe platform. Although the operating principle of this device is different to that we propose, as well as that demonstrated in [9], the tuning efficiency is of the order of $2 \mathrm{~K} / \mathrm{W}$ which is closer to that 
estimated for our own platform. Although the SiGe platform provides higher refractive index contrast than the GaAs/InGaP platform, it is also lower than that of the SOI platform, and the specific device was evaluated at $\lambda=7.7 \mu \mathrm{m}$ and thus required thicker claddings in order to avoid large optical overlap between the propagating mode and the heater that reduced the efficiency of the devices. Moreover, the thermal conductivity of $\mathrm{SiGe}$ is several orders of magnitude higher than that of $\mathrm{SiO}_{2}$.

A conservative design with large waveguide separation and large bend radii has been described in this study. The power consumption could be further reduced by making the spiral regions more compact.

\subsection{Extension to $15-16 \mu$ m operation}

This GaAs on InGaP spectrometer platform is expected to operate throughout most of the mid-infrared range up to a wavelength of $\sim 17 \mu \mathrm{m}$. In order for the platform to support operation at longer $\lambda$, the geometry of the layers has to be reoptimized. When re-designing a waveguide platform for operation at longer wavelengths, the increased optical losses due to tunnelling to the underlying substrate should be considered as well as the fundamental mode cut-off and compensated by re-optimising the core/cladding layer dimensions and the minimum bend radii. In order to produce a design for longer wavelengths and estimate new footprint and power consumption, optical and thermal simulations were performed at $15 \mu \mathrm{m}<\lambda<16 \mu \mathrm{m}$. Single mode operation was optimised at $\lambda=15 \mu \mathrm{m}$ and the optical losses were estimated at $\lambda=16 \mu \mathrm{m}$. Table 5 shows the optimised dimensions for low loss single mode operation as well as the thermal/power performance of the spectrometer at wavelengths between 15 and $16 \mu \mathrm{m}$. It is evident that the required thickness for the cladding layers is three times larger than in the case of $\lambda=$ $4 \mu \mathrm{m}$, which is expected as the mode size increases with increasing wavelength. The minimum bend radii required to mitigate the bend induced radiation losses is also larger than before, however, the footprint of the device remains the same since the spirals were already designed very conservatively for operation at wavelengths between 3 and $4 \mu \mathrm{m}$. As can be seen in Figure 10, the performance of the spectrometer significantly improves when a thicker lower cladding layer is introduced due to the enhanced thermal insulation between the heater and the substrate. Thus, as expected, the power efficiency of the device improves significantly (up to $\sim 29 \%$ ) with a similar transient response, when the device is optimised for operation at wavelengths between $15-16 \mu \mathrm{m}$.
Table 5. Estimated design and performance parameters at extended wavelength TE operation, a) Insulated boundary b) Heat sink boundary

\begin{tabular}{|cc|}
\hline $\begin{array}{c}\text { Waveguide core dimensions- } \\
\text { width } \times \text { thickness }(\mu \mathrm{m})\end{array}$ & $8 \times 7.5$ \\
\hline $\begin{array}{c}\text { Waveguide cladding } \\
\text { thickness }(\mu \mathrm{m})\end{array}$ & 15 \\
\hline Minimum bend radius $(\mu \mathrm{m})$ & 350 \\
\hline $\begin{array}{c}\text { Waveguide temperature rise } \\
(\mathrm{K})\end{array}$ & $\begin{array}{c}\text { a) } \sim 87.2 \\
\text { b) } \sim 88\end{array}$ \\
\hline Cross-talk temperature rise & a) $\sim 0.78$ \\
$(\mathrm{~K})$ & b) $\sim 0.01$ \\
\hline $\begin{array}{c}\text { Thermal response time (ms) } \\
\text { Max. power consumption }\end{array}$ & a) 18 \\
$(\mathrm{~W})$ & b) 11 \\
\hline Cooling time- $1 / \mathrm{e}^{2}$ of total & b) $\sim 29.1$ \\
excursion $(\mathrm{ms})$ & a) $\sim 1$ \\
\hline
\end{tabular}

\section{Conclusion}

An on-chip MIR thermo-optic spectrometer utilising a GaAs on InGaP waveguide layer structure has been designed and modelled. This study has focused on a device for operation between $\lambda=3$ and $4 \mu \mathrm{m}$ but this material platform is suitable for operation in any wavelength band between 1 and $17 \mu \mathrm{m}$. GaAs on InGaP is known for its high growth quality and low lattice mismatch. GaAs waveguides have shown very low propagation losses of less than $0.5 \mathrm{~dB} \mathrm{~cm}^{-1}$ at $\lambda=1310-1550$ nm. Both semiconductor materials offer wide MIR transmission extending over the molecular fingerprint absorption region. Through our simulation results, we show that with proper structural design, the platform can be tailored to provide very low optical losses for single mode operation. However, the low refractive index contrast between the waveguide and the cladding results in dimensionally large devices when compared to other platforms such as silicon-oninsulator (SOI). Thermal modelling revealed that the layer dimensions as well as the thermal insulation of the device can greatly affect its performance. In summary, with the appropriate design, the proposed device can achieve a good performance which is reflected by the acceptable power needs as well as the potential for extension to much longer wavelengths in the MIR. That makes it a very promising platform for the realisation of an ultra-portable MIR spectrometer. In addition, its use is not only limited to spectroscopy applications, but it can also be exploited in many other applications such as MIR modulators and phase shifters.

\section{Acknowledgments}

This work was supported by "The Future Photonics Hub" (EPSRC grant EP/N00762X/1). The authors would like to acknowledge Professor Jon Heffernan and Dr. Kris Groom from the University of Sheffield, for useful discussions regarding the material platform used in this study.

The data for this work is accessible through the University of Southampton Institutional Research Repository (https://doi.org/10.5258/SOTON/D1335). 


\section{References}

[1] T. Scharf, D. Briand, S. Bühler, O. Manzardo, H.P. Herzig, N.F. de Rooij, Miniaturized Fourier transform spectrometer for gas detection in the MIR region, Sensors and Actuators B: Chemical, 147 (2010) 116-121.

[2] Ferhanoglu O, Seren H, Lüttjohann S and Urey H, Lamellar grating optimization for miniaturized fourier transform spectrometers, Opt Express, 1721289 (2009).

[3] Manzardo O, Michaely R, Schädelin F, Noell W, Overstolz T, De Rooij $\mathrm{N}$ and Herzig H, Miniature lamellar grating interferometer based on silicon technology, Opt Lett, 29 (2004) 1437.

[4] H.R. Seren, S. Holmstrom, N.P. Ayerden, J. Sharma, H. Urey, Lamellar-Grating-Based MEMS Fourier Transform Spectrometer, Journal of Microelectromechanical Systems, 21 (2012) 331-339. [5] Y.-M. Lee, M. Toda, M. Esashi, T. Ono, Micro wishbone interferometer for Fourier transform infrared spectrometry, Journal of Micromechanics and Microengineering, 21 (2011) [6] J. Li, D.F. Lu, Z.M. Qi, Miniature Fourier transform spectrometer based on wavelength dependence of half-wave voltage of a $\mathrm{LiNbO}(3)$ waveguide interferometer, Opt Lett, 39 (2014) 3923-3926.

[7] J. Loridat, S. Heidmann, F. Thomas, G. Ulliac, N. Courjal, A Morand, G. Martin, All Integrated Lithium Niobate Standing Wave Fourier Transform Electro-Optic Spectrometer, Journal of Lightwave Technology, 36 (2018) 4900-4907.

[8] G.G. Bentini, M. Bianconi, A. Cerutti, M. Chiarini, G. Pennestrì, C. Sada, N. Argiolas, M. Bazzan, P. Mazzoldi, Integrated Mach-Zehnder micro-interferometer on LiNbO3, Optics and Lasers in Engineering, 45 (2007) 368-372.

[9] M. Souza, A. Grieco, N.C. Frateschi, Y. Fainman, Fourier transform spectrometer on silicon with thermo-optic non-linearity and dispersion correction, Nat Commun, 9 (2018) 665.

[10] A.Z. Subramanian, E. Ryckeboer, A. Dhakal, F. Peyskens, A. Malik, B. Kuyken, H. Zhao, S. Pathak, A. Ruocco, A. De Groote, P. Wuytens, D. Martens, F. Leo, W. Xie, U.D. Dave, M. Muneeb, P. Van Dorpe, J. Van Campenhout, W. Bogaerts, P. Bienstman, N. Le Thomas, D. Van Thourhout, Z. Hens, G. Roelkens, R. Baets, Silicon and silicon nitride photonic circuits for spectroscopic sensing on-a-chip [Invited], Photonics Research, 3 (2015).

[11] D.M. Kita, B. Miranda, D. Favela, D. Bono, J. Michon, H. Lin, T. Gu, J. Hu, High-performance and scalable on-chip digital Fourier transform spectroscopy, Nat Commun, 9 (2018) 4405 [12] J. Haas, B. Mizaikoff, Advances in Mid-Infrared Spectroscopy for Chemical Analysis, Annu Rev Anal Chem (Palo Alto Calif), 9 (2016) 45-68.

[13] R. Crocombe, EXPRESS: Portable Spectroscopy, Appl Spectrosc, (2018) 3702818809719

[14] J.M. Ramirez, Q. Liu, V. Vakarin, J. Frigerio, A. Ballabio, X. Le Roux, D. Bouville, L. Vivien, G. Isella, D. Marris-Morini, Graded SiGe waveguides with broadband low-loss propagation in the mid infrared, Opt Express, 26 (2018) 870-877.

[15] P.M. C. Grillet, B. Luther-Davies, D. Hudson, C. Monat, S Madden, D. J. Moss, M. Brun,, S.O.a.S.N. P. Labeye, Low loss $\mathrm{SiGe}$ waveguides in the MID-IR, Conference on Lasers and Electro-Optics Europe, Munich, Germany, 2013.

[16] Q. Liu, J.M. Ramirez, V. Vakarin, X. Le Roux, C. AlonsoRamos, J. Frigerio, A. Ballabio, E. Talamas Simola, D. Bouville, L. Vivien, G. Isella, D. Marris-Morini, Integrated broadband dualpolarization Ge-rich SiGe mid-infrared Fourier-transform spectrometer, Opt Lett, 43 (2018) 5021-5024.

[17] M. Montesinos-Ballester, Q. Liu, V. Vakarin, J.M. Ramirez, C. Alonso-Ramos, X.L. Roux, J. Frigerio, A. Ballabio, E. Talamas, L. Vivien, G. Isella, D. Marris-Morini, On-chip Fouriertransform spectrometer based on spatial heterodyning tuned by thermo-optic effect, Sci Rep, 9 (2019) 14633

[18] K. Gallacher, R.W. Millar, U. Griskeviciute, L. Baldassarre, M. Sorel, M. Ortolani, D.J. Paul, Low loss Ge-on-Si waveguides operating in the 8-14 microm atmospheric transmission window, Opt Express, 26 (2018) 25667-25675.

[19] Byun Y, Park K, Kim S, Choi S and Lim T, Single-mode GaAs/AlGaAs W waveguides with a low propagation loss, Appl Opt, 35 (1996) 928.

[20] Inoue H, Hiruma K, Ishida K, Asai T and Matsumura H, Low loss GaAs optical waveguides, IEEE T Electron Dev, 32 (1985) 2662-2668.

[21] A.D. Ferguson, S. Iezekiel, Y. Zhou, C.M. Snowden, A. Kuver, J.M. Heaton, Low-loss, single-mode GaAs/AlGaAs waveguides with large core thickness, IEE Proceedings Optoelectronics, 153 (2006) 51-56.

[22] Baojun L and Chua S J, Optical Switches: Materials and Design, WP Publishing, Cambridge, 2010.

[23] Byun Y T, Kim J S, Kim S H and chang Yi J, Linear ElectroOptic Coefficient of $\mathrm{GaAs} / \mathrm{Al}_{0.4} \mathrm{Ga}_{06} \mathrm{As}$ Phase Modulator, J Korean Phys Soc, 45 (2004) 1162-1164

[24] Jaeger A F N, Rahmatian F, James R and Berolo E, An AlGaAs/GaAs Mch Zehnder Modulator Using Slow Wave Coplanar Electrodes, 1998 IEEE Canadian Conference on Electrical and Computer Engineering (1998) 802-805.

[25] Shin J and Dagli N, Ultra-low voltage GaAs/AlGaAs MachZehnder intensity modulators, IEEE Avionics, Fiber-Optics and Photonics Technology Conference (2008).

[26] Weber M J, Handbook of Optical Materials, CRC Press, New York, 2003.

[27] R. Ferrini, G. Guizzetti, M. Patrini, A. Parisini, L. Tarricone, B. Valenti, Optical functions of InGaP/GaAs epitaxial layers from 0.01 to $5.5 \mathrm{eV}$, The European Physical Journal B - Condensed Matter, 27 (2002) 449-458

[28] Camargo E, Chong H and De La Rue R, 2D Photonic crystal thermo-optic switch based on AlGaAs/GaAs epitaxial structure, Opt Express 12 (2004) 588.

[29] Rathi M, Dutta P, Zheng N, Yao Y, Gao Y, Sun S,

Khadimallah A, Thomas M, Asadirad M, Ahrenkiel P, Ryou J and Selvamanickam V, AlGaAs/GaAs DH and $\mathrm{InGaP} / \mathrm{GaAs} \mathrm{DH}$ grown by MOCVD on flexible metal substrates, IEEE 43rd Photovoltaic Specialists Conference (PVSC) (2016).

[30] Schubert M, Gottschalch V, Herzinger C, Yao H, Snyder P and Woollam J, Optical constants of GaxIn1-xP lattice matched to GaAs J. Appl. Phys. 77 (1995) 3416-3419.

[31] Refractive index of GaAs

https://www.batop.de/information/n_GaAs.html, 2019 (accessed 15 December 2019)

[32] S. Sokolov, J. Lian, S. Combrie, A. De Rossi, A.P. Mosk, Measurement of the linear thermo-optical coefficient of $\mathrm{Ga}_{0.51} \mathrm{In}_{0.49} \mathrm{P}$ using photonic crystal nanocavities, Appl Opt, 56 (2017) 3219-3222

[33] F.G. Della Corte, G. Cocorullo, M. Iodice, I. Rendina, Temperature dependence of the thermo-optic coefficient of InP, $\mathrm{GaAs}$, and $\mathrm{SiC}$ from room temperature to $600 \mathrm{~K}$ at the wavelength of $1.5 \mu \mathrm{m}$, Applied Physics Letters, 77 (2000) 1614-1616. [34] M. Nedeljkovic, S. Stankovic, C.J. Mitchell, A.Z. Khokhar, S.A. Reynolds, D.J. Thomson, F.Y. Gardes, C.G. Littlejohns, G.T. Reed, G.Z. Mashanovich, Mid-Infrared Thermo-Optic Modulators in SoI, IEEE Photonics Technology Letters, 26 (2014) 1352-1355. [35] M. Sieger, F. Balluff, X. Wang, S.S. Kim, L. Leidner, G. Gauglitz, B. Mizaikoff, On-chip integrated mid-infrared GaAs/AlGaAs Mach-Zehnder interferometer, Anal Chem, 85 (2013) 3050-3052

[36] Barnes W, Dereux A and Ebbesen T, Surface plasmon subwavelength optics, Nature, 424 (2003) 824-830. [37] Murugan G, Mittal V, Craig C, Hewak D and Wilkinson J, Chalcogenide waveguides for mid-infrared biomedical sensing applications, Photonics North, Ottawa, ON (2015).

[38] Sun P and Reano R, Submilliwatt thermo-optic switches using free-standing silicon-on-insulator strip waveguides, Opt. Express 18 (2010) 8406.

[39] M. Baroncini, P. Placidi, G.C. Cardinali, A. Scorzoni, Thermal characterization of a microheater for micromachined gas sensors, Sensors and Actuators A: Physical, 115 (2004) 8-14. 
[40] A. VanHorn, W. Zhou, Design and optimization of a high

temperature microheater for inkjet deposition, The International Journal of Advanced Manufacturing Technology, 86 (2016) 3101 -

3111.

[41] V. Palankouski, R. Schultheis, S. Selberherr, Simulation of power heterojunction bipolar transistors on gallium arsenide,

IEEE Transactions on Electron Devices, 48 (2001) 1264-1269. 